\title{
Multiple genes encoding coiled-coil domain-containing proteins are simultaneously differentially expressed in metastasis to the lung in a HER2+ mouse model of metastatic breast cancer
}

\author{
Shahan Mamoor, MS1 \\ 1Thomas Jefferson School of Law \\ San Diego, CA 92101 \\ mamoorsk@tjsl.edu
}

Breast cancer is one of the leading causes of deaths from cancers for women in the United States and accounts for 42,000 deaths each year (1). Metastasis, spread of the disease from the breast to a foreign tissue site like the brain and lungs, is the major cause of mortality for women with breast cancer (2). Targeted therapeutics aimed at halting, slowing or reversing metastasis requires understanding what the major differences are between the primary tumor and the metastatic tumor at the level of gene expression. We mined a public dataset containing transcriptome data from a murine model of HER2+ breast cancer that mimics the human disease including spread of the disease to the lungs (3), to understand the major transcriptional differences between breast cancers as they arise and the metastases that they generate. We found that five genes that encode the coiled-coil domain containing (CCDC) family of proteins were among the most significantly differentially expressed genes when comparing the global gene expression profiles of tissue from the primary breast tumor to tissues from lung metastases. A single-nucleotide polymorphism at a coiled-coil domaincontaining gene locus has been implicated by GWAS as a contributor to human breast cancer $(4,5)$ and these data provide blind confirmation of their importance at the systems level, supporting the concept that targeting of CCDC proteins may be of therapeutic relevance in human metastatic breast cancer.

Keywords: Coiled-coil domain-containing proteins, CCDC, HER2+ breast cancer, metastatic breast cancer, lung metastases, differential gene expression, systems oncology, comparative transcriptomics of primary and metastatic tumors. 


\section{Introduction}

Breast cancer affects 252,000 women every year in the United States, and women living in the United States have a $12 \%$ chance of developing breast cancer sometime during their life (1). Over 40,000 of these women will die from the disease each year (1). Though the 5-year survival rate for women with localized disease is $99 \%$, and $85 \%$ for women with disease that has spread to the lymph nodes, it is $27 \%$ for women with disease that has metastasized, or spread to distant organs such as the lung (6). Novel therapeutic targets to halt or delay metastasis are of critical need.

Understanding how the breast cancer transcriptome is altered relative to healthy, noncancerous tissue can enhance efforts towards the discovery of novel therapeutic targets (7). Describing the transcriptional changes between primary tumor and metastasis are similarly crucial $(8,9,10)$. The aim of this analysis was to describe the most significant differences between the transcriptional profiles of metastases to the primary tumors from which they originate. We mined a published dataset from a study utilizing the HER2+ BALB-NeuT mouse model of breast cancer in conjunction with microarray gene expression profiling (3). Salient features of this BALB-NeuT model include development of an independent tumor in each of 10 mammary glands and metastasis to the lung $(11,12)$.

\section{Methods}

GEO2R was utilized for this differential gene expression analysis, in conjunction with dataset GSE68683 (3) with platform GPL13188. Data from ten primary tumor samples, derived from the center of the tumor (as opposed to the tumor margins) were compared to data from lung metastasis samples. Log transformation of data was set to "Auto-detect", and the $p$ value adjustment was set to "Benjamini-Yekutieli". This $p$-value refers to the significance of the differential expression at the global level, when comparing groups (primary tumors versus metastases). The "Submitter supplied" category of platform annotation was used to identify 
names of genes from which the differentially transcribed RNAs correspond to. To determine the statistical significance of the difference in mean RNA expression values between tumor and metastatic groups for each differentially expressed CCDC gene, a two-tailed, unpaired t-test with Welch's correction was performed using PRISM (8.1.2) (227).

\section{Results}

Five genes of the coiled-coil domain-containing family are differentially expressed at the RNA level in an autochthonous murine model of HER2+ breast cancer in Balb-NeuT mice

To understand the transcriptional changes between primary tumors of the breast to the metastases that they generate, we performed global differential gene expression profiling, comparing published transcriptome data from two types of tumor tissues from a HER2+ breast cancer mouse model (3): ten breast tumors compared to seven lung metastases. These analyses revealed that among the genes whose expression was most different between the primary breast tumor and the lung metastases that they generated were five independent transcripts encoded by Ccdc genes: Ccdc93, Ccdc49, Ccdc100, Ccdc125, and Ccdc97. Out of 36009 total transcripts that were detected by the microarray data analyzed in this study, Ccdc93 ranked 34 most differentially expressed $(p=0.0002933)$, Ccdc49 ranked 175 ( $p=$ 0.00184947), Ccdc100 ranked $235(p=0.00274248)$, Ccdc125 ranked $237(p=0.00275701)$, and Ccdc97 ranked $239(p=0.00279693)($ Table 1).

Next, we retrieved the expression values for each tissue sample representing primary breast tumor and lung metastasis, and compared the significance of the mean values between each group using a two-tailed, unpaired t-test with Welch's correction. For Ccdc93, Ccdc49, and Ccdc125, the expression of the gene was significantly lower in the lung metastases (Figure 1; Ccdc93: $p=0.0017$, Ccdc49: $p=0.0063$, and Ccdc125, $p=0.0046)$. For Ccdc97 and Ccdc100, however, the expression of the gene was significantly higher in the lung metastasis tissues (Figure 1; Ccdc97, $p=0.0076$ and Ccdc100, $p=0.0255$ ). 


\section{Discussion}

Breast cancer is the second leading cause of mortality from cancer for women in the United States (1). Breast cancer results in 42,000 deaths each year in the United States, and metastasis, spread of the disease from the breast to a foreign site such as the brain, lungs and bones is the most common reason leading to mortality (2). Standard chemotherapies target all cells, with specificity generally being conferred by the rate at which cells divide, and not any other intrinsic property of the cancer cell, resulting in toxicities $(13,14)$. There is a real need for targeted therapies for the treatment of metastatic breast cancers, to halt their progression or to reverse its course. Systems-level analysis of cancers have the potential to reveal the most fundamental biology underlying the mechanisms by which cells transform into tumor cells, and by which tumors result in the colonization of a foreign tissue with metastasis $(15,16,17,18)$.

Development of targeted therapies to treat metastatic breast cancers requires an understanding of the pathways and transcriptional networks that breast cancers use to metastasize, whether it be in the maintenance of the long-sought after tumor stem cell (19-22), in the breach of the lamina surrounding the primary tumor $(23,24)$, in the extravasation out of and intravasation into the foreign site $(25,26)$, or the homing or colonization of the metastatic site $(27,28)$. Differential gene expression of microarray or RNA-sequencing data from tumor cells or tissue compared to the cell or tissue of origin, or from the primary tumor compared to the metastatic tumor, can provide this information in an unbiased, global manner.

Here, we performed a global differential gene expression analysis by comparing the transcriptomes of ten breast tumors to seven lung metastasis, using microarray data from a published data set (3). This analysis revealed that five unique genes of the Ccdc family of coiled-coil domain containing proteins were among the most differentially expressed genes in the lung metastases of mice engineered to develop a HER2+ breast cancer. 
While faithful mouse models of human cancers can be helpful and relevant in preclinical assessment of pathway perturbation or novel compounds targeting these pathways, translation of findings from mouse models at the level of target discovery can be frustrating as there may be species-divergent mechanisms governing this biology. In this case, however, human genetics provide evidence that these findings in mice are not species-divergent but instead strong confirmation that Ccdc proteins are relevant pathways in target discovery and development of novel therapeutics for human breast cancer. Systems-level analysis (29) can be performed at the level of the protein by mass spectrometric proteomic analysis (30), at the level of the transcript as was performed here by comparative differential gene expression profiling of transcriptome data obtained using microarray $(16,17)$, or at the level of the genome, whether in the whole-genome identification of epigenetic modifications such as DNA methylation (31) or in single-base pair differences, identified by comparing single-nucleotide polymorphisms (SNPs) by genome-wide association studies of sequenced patient DNA $(4,5)$.

One such GWAS study, compared the genetic information at over 600,000 SNPs in 1505 cases of breast cancer versus 1522 controls in a Chinese cohort identified a SNP at the 6q25.1 locus, upstream of Esr1, the estrogen receptor, as being a causal variant that significantly associated with risk for development of breast cancer, This study then analyzed 1591 cases and 1466 controls in a European cohort and found that the 6q25.1 locus again significantly associated with the risk of developing breast cancer, thus the C6orf97 locus (CCDC170) was implicated as being significantly associated with the development of breast cancer in humans (4). 
A second study genotyped 582,886 SNPs in 3659 cases versus 4,897 controls and again identified this single nucleotide polymorphism at the 6q25.1 locus as being a significant factor in the susceptibility to breast cancer (5). Thus, systems-level analysis of the genetic variation in patients with breast cancer, from two separate genome-wide analyses, has identified a Ccdc gene as being relevant for understanding the biology of tumorigenesis and/or in the development of therapeutics to modulate the gene product from this locus. Another mechanistic study showed that CCDC170, by its localization in the cell at the Golgi network, could exert control over cell migration by interactions with the Golgi microtubule cytoskeleton, modulating the inner and outer edge of the cell membrane and thus its directionality of movement (32).

Moreover, one other study examined the specific effects of the association of the SNP identified by these GWAS studies, rs2046210, and its linkage disequilibrium (significant genetic association with) with the highly proliferative characteristics and worse relapse free survival, and gene expression levels of Ccdc170 and two other C6ORF genes, C6ORF211 and C6ORF:RMND1. Only C6ORF97/Ccdc170, of the three C6ORF genes examined, showed significant association with highly proliferative characteristics and worse relapse free survival in fact, the other two C6ORFs showed negative correlation, in fact. C6ORF97 expression significantly correlated with worse prognostic value in the luminal B subtype of breast cancer. These data demonstrated that C6ORF97, and not another transcript that is produced at the C6ORF locus, was responsible for the genetic association seen in GWAS studies that detected a causal variant SNP at the ESR1-C6ORF97 locus, and that expression of C6ORF97 (also known as Ccdc170) was significantly associated with aggressive nature of the disease manifested by worse progression free survival and a highly proliferative characteristic of the disease (Ki67 staining, a nuclear antigen associated with proliferation) (33). 
With respect to other cancers, another Ccdc family protein, Ccdc98 was has been shown, at the protein level to interact with the breast cancer gene-1, BRCA1 in lung cancers (34). Interaction of Ccdc98 with BRCA1 was important for activation of DNA damage response in response to ionizing radiation and cell cycle damage control at the interface of the G2 and M phases. In another study of non-small cell lung cancer, Ccdc106 was important for the proliferative capacity of lung cancer cell lines in vitro and in vivo, enhancing expression of the cell cycle genes Cyclin A2 and Cyclin B1, and promoted Akt phosphorylation (35). In a third study of lung cancers, expression of Ccdc85b was significantly associated with lymph node invasion and metastasis, and knockdown of Ccdc85b in the A549 lung cancer cell line the invasion and migration capacity of these lung cancer cells in vitro (36). Ccdc85b in this context was found to phosphorylate Akt as well as Gsk3ß, as well as up-regulate the expression of $\boldsymbol{\beta}$ catenin and the Wnt pathway targets myc, Cyclin D1 and the matrix metalloproteinase MMP7 (36).

Together, these systems GWAS studies, along with a mechanistic study demonstrating an effect of the gene product at the Ccdc170 locus on cell migration and movement, as well as three other studies showing that the gene product of three other Ccdc family proteins Ccdc98, Ccdc106, and Ccdc85b played a role in the progression of non-small cell lung cancers, interacting with the major breast cancer susceptibility gene Brca1 (34), controlling the proliferation of a lung cancer cell line (35), and influencing the invasion and migration capacity of a lung cancer cell line (36), supports the assertion that the findings from this analysis here are of relevance to the conclusion that the targeted study of Ccdc proteins in causing or contributing to the processes of tumor formation and metastasis in human metastatic breast cancer should be prioritized. 
Breast cancer is a leading cause of cancer death in the United States and metastasis is what causes this death (1). It is imperative to understand the transcriptional behavior of metastases and how they differ from the tumors they arise from. In this study, we asked what the major transcriptional differences were between tumors of the breast and the lung metastases that they generate by analyzing a public dataset of tumor and metastasis tissue from a mouse model of HER2+ metastatic breast cancer. Global differential gene expression profiling showed that five of the two hundred and fifty most differentially expressed genes when comparing tumors and metastases in this model were from the Ccdc family of coiled-coil domain containing proteins.

Together with prior systems-level GWAS data implicating a SNP proximal to Ccdc170 and its genetic association with the risk for developing breast cancer in multiple cohorts $(4,5$, 31), and with mechanistic studies showing that Ccdc170 could influence cell migration and motility by interacting with the microtubule cytoskeleton at the Golgi complex (32), our results here are clear demonstration that Ccdc170 and the Ccdc family of proteins should be prioritized for understanding of the biological pathways that cause breast cancer in humans, that lead to the metastasis that cause their deaths, and for the development of novel therapeutics to treat this malady. 


\section{References}

1. DeSantis, C.E., Ma, J., Goding Sauer, A., Newman, L.A. and Jemal, A., 2017. Breast cancer statistics, 2017, racial disparity in mortality by state. CA: a cancer journal for clinicians, 67(6), pp.439-448.

2. Mehlen, Patrick, and Alain Puisieux. "Metastasis: a question of life or death." Nature Reviews Cancer 6.6 (2006): 449.

3. Hosseini, H., Obradović, M.M., Hoffmann, M., Harper, K.L., Sosa, M.S., Werner-Klein, M., Nanduri, L.K., Werno, C., Ehrl, C., Maneck, M. and Patwary, N., 2016. Early dissemination seeds metastasis in breast cancer. Nature, 540(7634), p.552.

4. Zheng, W., Long, J., Gao, Y.T., Li, C., Zheng, Y., Xiang, Y.B., Wen, W., Levy, S., Deming, S.L., Haines, J.L. and Gu, K., 2009. Genome-wide association study identifies a new breast cancer susceptibility locus at 6q25. 1. Nature genetics, 41(3), p.324.

5. Turnbull, C., Ahmed, S., Morrison, J., Pernet, D., Renwick, A., Maranian, M., Seal, S., Ghoussaini, M., Hines, S., Healey, C.S. and Hughes, D., 2010. Genome-wide association study identifies five new breast cancer susceptibility loci. Nature genetics, 42(6), p.504.

6. ACS Cancer Facts \& Figures 2019, https://www.cancer.net/cancer-types/breast-cancermetastatic/statistics.

7. Sgroi, D.C., Teng, S., Robinson, G., LeVangie, R., Hudson, J.R. and Elkahloun, A.G., 1999. In vivo gene expression profile analysis of human breast cancer progression. Cancer research, 59(22), pp.5656-5661.

8. Zhao, H., Li, Y., Wang, S., Yang, Y., Wang, J., Ruan, X., Yang, Y., Cai, K., Zhang, B., Cui, P. and Yan, J., 2014. Whole transcriptome RNA-seq analysis: tumorigenesis and metastasis of melanoma. Gene, 548(2), pp.234-243.

9. Zhang, J., Huang, J.Y., Chen, Y.N., Yuan, F., Zhang, H., Yan, F.H., Wang, M.J., Wang, G., Su, M., Lu, G. and Huang, Y., 2015. Whole genome and transcriptome sequencing of matched primary and peritoneal metastatic gastric carcinoma. Scientific reports, 5, p.13750.

10. Puram, S.V., Tirosh, I., Parikh, A.S., Patel, A.P., Yizhak, K., Gillespie, S., Rodman, C., Luo, C.L., Mroz, E.A., Emerick, K.S. and Deschler, D.G., 2017. Single-cell transcriptomic analysis of primary and metastatic tumor ecosystems in head and neck cancer. Cell, 171(7), pp. 1611-1624.

11. lezzi, M., Calogero, R.A., Spadaro, M., Musiani, P., Forni, G. and Cavallo, F., 2012. BALBneuT Female Mice as a Dynamic Model of Mammary Cancer. Translational Animal Models in Drug Discovery and Development, p.139.

12. Conti, L., Ruiu, R., Barutello, G., Macagno, M., Bandini, S., Cavallo, F. and Lanzardo, S., 2014. Microenvironment, oncoantigens, and antitumor vaccination: lessons learned from BALB-neuT mice. BioMed research international, 2014.

13. DeVita, V.T. and Chu, E., 2008. A history of cancer chemotherapy. Cancer research, 68(21), pp.8643-8653. 
14. Chabner, B.A. and Roberts Jr, T.G., 2005. Chemotherapy and the war on cancer. Nature Reviews Cancer, 5(1), p.65.

15. Rhodes, D.R. and Chinnaiyan, A.M., 2005. Integrative analysis of the cancer transcriptome. Nature genetics, 37(6s), p.S31.

16. Zhang, J., Huang, J.Y., Chen, Y.N., Yuan, F., Zhang, H., Yan, F.H., Wang, M.J., Wang, G., Su, M., Lu, G. and Huang, Y., 2015. Whole genome and transcriptome sequencing of matched primary and peritoneal metastatic gastric carcinoma. Scientific reports, 5, p.13750.

17. Puram, S.V., Tirosh, I., Parikh, A.S., Patel, A.P., Yizhak, K., Gillespie, S., Rodman, C., Luo, C.L., Mroz, E.A., Emerick, K.S. and Deschler, D.G., 2017. Single-cell transcriptomic analysis of primary and metastatic tumor ecosystems in head and neck cancer. Cell, 171(7), pp. 1611-1624.

18. Cieślik, M. and Chinnaiyan, A.M., 2018. Cancer transcriptome profiling at the juncture of clinical translation. Nature Reviews Genetics, 19(2), pp.93-109.

19. Ponti, D., Costa, A., Zaffaroni, N., Pratesi, G., Petrangolini, G., Coradini, D., Pilotti, S., Pierotti, M.A. and Daidone, M.G., 2005. Isolation and in vitro propagation of tumorigenic breast cancer cells with stem/progenitor cell properties. Cancer research, 65(13), pp. 5506-5511.

20. Charafe-Jauffret, E., Ginestier, C., lovino, F., Wicinski, J., Cervera, N., Finetti, P., Hur, M.H., Diebel, M.E., Monville, F., Dutcher, J. and Brown, M., 2009. Breast cancer cell lines contain functional cancer stem cells with metastatic capacity and a distinct molecular signature. Cancer research, 69(4), pp.1302-1313.

21. Balic, M., Lin, H., Young, L., Hawes, D., Giuliano, A., McNamara, G., Datar, R.H. and Cote, R.J., 2006. Most early disseminated cancer cells detected in bone marrow of breast cancer patients have a putative breast cancer stem cell phenotype. Clinical cancer research, 12(19), pp.5615-5621.

22. Cordenonsi, M., Zanconato, F., Azzolin, L., Forcato, M., Rosato, A., Frasson, C., Inui, M., Montagner, M., Parenti, A.R., Poletti, A. and Daidone, M.G., 2011. The Hippo transducer TAZ confers cancer stem cell-related traits on breast cancer cells. Cell, 147(4), pp.759-772.

23. Muschler, J. and Streuli, C.H., 2010. Cell-matrix interactions in mammary gland development and breast cancer. Cold Spring Harbor perspectives in biology, 2(10), p.a003202.

24. Pitelka, D.R., Hamamoto, S.T. and Taggart, B.N., 1980. Basal lamina and tissue recognition in malignant mammary tumors. Cancer research, 40(5), pp.1600-1611.

25. Peng, F., Setyawati, M.I., Tee, J.K., Ding, X., Wang, J., Nga, M.E., Ho, H.K. and Leong, D.T., 2019. Nanoparticles promote in vivo breast cancer cell intravasation and extravasation by inducing endothelial leakiness. Nature nanotechnology, 14(3), pp.279-286.

26. Mego, M., Mani, S.A. and Cristofanilli, M., 2010. Molecular mechanisms of metastasis in breast cancer-clinical applications. Nature reviews Clinical oncology, 7(12), p.693. 
27. Liapis, H., Flath, A. and Kitazawa, S., 1996. Integrin alpha $V$ beta 3 expression by boneresiding breast cancer metastases. Diagnostic molecular pathology: the American journal of surgical pathology, part B, 5(2), pp.127-135.

28. Moore, M.A., 2001. The role of chemoattraction in cancer metastases. Bioessays, 23(8), pp. 674-676.

29. Kitano, H., 2002. Systems biology: a brief overview. science, 295(5560), pp.1662-1664.

30. Yanagisawa, K., Shyr, Y., Xu, B.J., Massion, P.P., Larsen, P.H., White, B.C., Roberts, J.R., Edgerton, M., Gonzalez, A., Nadaf, S. and Moore, J.H., 2003. Proteomic patterns of tumour subsets in non-small-cell lung cancer. The Lancet, 362(9382), pp.433-439.

31. Jones, P.A. and Baylin, S.B., 2007. The epigenomics of cancer. Cell, 128(4), pp.683-692.

32. Jiang, P., Li, Y., Poleshko, A., Medvedeva, V., Baulina, N., Zhang, Y., Zhou, Y., Slater, C.M., Pellegrin, T., Wasserman, J. and Lindy, M., 2017. The protein encoded by the CCDC170 breast cancer gene functions to organize the golgi-microtubule network. EBioMedicine, 22, pp.28-43.

33. Yamamoto-Ibusuki, M., Yamamoto, Y., Fujiwara, S., Sueta, A., Yamamoto, S., Hayashi, M., Tomiguchi, M., Takeshita, T. and Iwase, H., 2015. C6ORF97-ESR1 breast cancer susceptibility locus: influence on progression and survival in breast cancer patients. European Journal of Human Genetics, 23(7), p.949.

34. Kim, H., Huang, J. and Chen, J., 2007. CCDC98 is a BRCA1-BRCT domain-binding protein involved in the DNA damage response. Nature structural \& molecular biology, 14(8), p.710.

35. Zhang, X., Zheng, Q., Wang, C., Zhou, H., Jiang, G., Miao, Y., Zhang, Y., Liu, Y., Li, Q., Qiu, X. and Wang, E., 2017. CCDC106 promotes non-small cell lung cancer cell proliferation. Oncotarget, 8(16), p.26662.

36. Feng, Y., Gao, Y., Yu, J., Jiang, G., Zhang, X., Lin, X., Han, Q., Rong, X., Xu, H., Li, Q. and Qiu, X., 2019. CCDC85B promotes non-small cell lung cancer cell proliferation and invasion. Molecular carcinogenesis, 58(1), pp.126-134. 

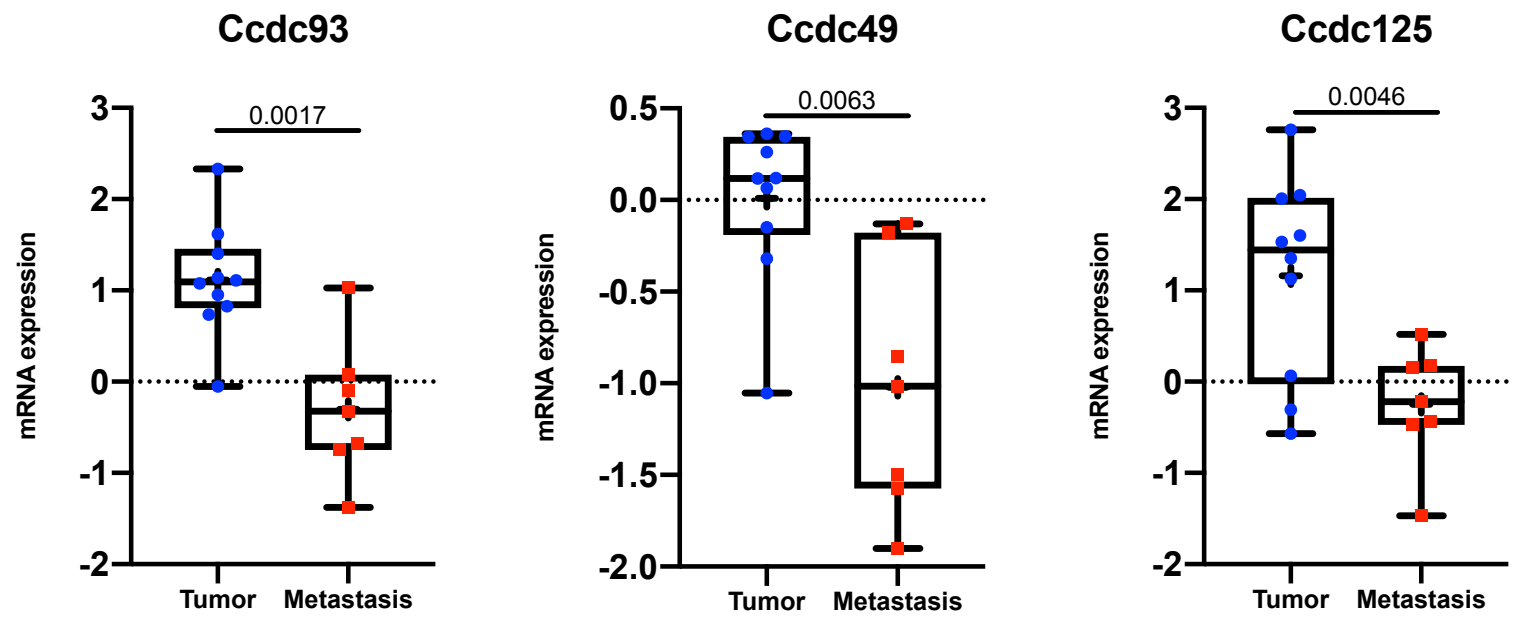

Ccdc97

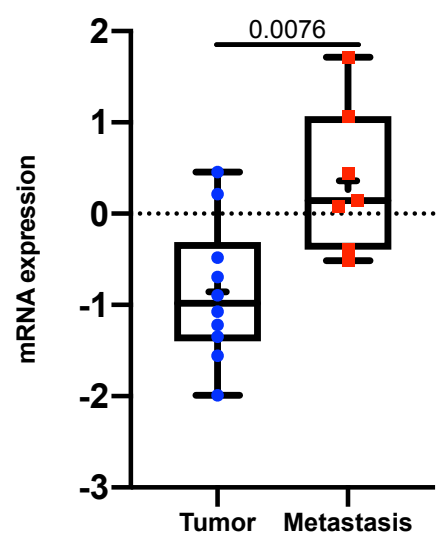

Ccdc100

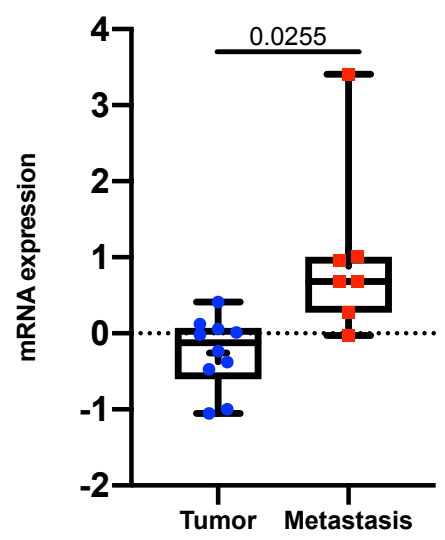

Figure 1: Five genes that encode coiled-coil domain-containing proteins are simultaneously transcriptionally perturbed in the metastatic transcriptome of a mouse model of HER2+ breast cancer.

The mRNA expression level of each differentially expressed Ccdc transcript is graphically depicted by box and whisker plot, with all points shown in color, the mean value shown as a (+) sign, and error bars extending from minimum to maximum value. A statistical test to compare the significance of difference between expression values between tumor and metastatic groups was performed by a two-tailed unpaired t-test with Welch's correction,; $p$-value shown above each graph. Primary breast tumors: $n=10$; lung metastases, $n=7$. 
Table 1

\begin{tabular}{|c|c|c|c|c|c|}
\hline Name & Rank (out of 36009) & $\underline{\text { ID }}$ & p-value & $\underline{\mathbf{t}}$ & $\underline{\mathbf{B}}$ \\
\hline Ccdc93 & 34 & 21975 & 0.0002933 & -4.2550896 & 0.1427 \\
\hline Ccdc49 & 175 & 30517 & 0.00184947 & -3.5130364 & -1.2882 \\
\hline Ccdc100 & 235 & 30206 & 0.00274248 & 3.3512629 & -1.5961 \\
\hline Ccdc125 & 237 & 32513 & 0.00275701 & -3.3490816 & -1.6003 \\
\hline Ccdc97 & 239 & 822 & 0.00279693 & 3.3431468 & -1.6115 \\
\hline
\end{tabular}

Table 1: Global differential gene expression analysis of tumors from a HER2+ murine model of breast cancer identifies five coiled coil domain-containing proteins as the most differentially expressed genes in the lung metastatic transcriptome.

Shown is the rank out of 36009 total transcripts detected, name of the gene, NCBI ID, $p$-value with respect to differential expression compared to all other transcripts detected, and two statistics, $t$ and $B$ : $t$ is a moderated $t$-statistic with respect to the expression between the two groups compared (tumor and metastasis), while B is a log-odds calculation that the gene is differentially expressed. 